\title{
Nonlinear Localized Waves in a Periodic Medium
}

\author{
Andrey A. Sukhorukov and Yuri S. Kivshar \\ Nonlinear Physics Group, Research School of Physical Sciences and Engineering, Australian National University, \\ Canberra ACT 0200, Australia
}

(Received 31 October 2000; published 1 August 2001)

\begin{abstract}
We analyze the existence and stability of nonlinear localized waves in a periodic medium described by the Kronig-Penney model with a nonlinear defect. We demonstrate the existence of a novel type of stable nonlinear band-gap localized state, and also reveal a generic physical mechanism of the oscillatory wave instabilities associated with the band-gap resonances.
\end{abstract}

DOI: 10.1103/PhysRevLett.87.083901

Wave propagation in periodic media is associated with many interesting physical phenomena [1]. Modern technology allows us to create different kinds of macroand mesoscopic periodic and layered structures such as semiconductor superlattices and heterostructures, magnetic multilayers possessing the giant magnetoresistance, multiple-quantum-well structures, optical waveguide arrays, photonic band-gap materials, etc. The main feature of different periodic structures (which follows from the classical Floquet-Bloch theory) is the existence of frequency band gaps (or stop bands) where linear waves undergo Bragg reflection from the periodic structure [1]. However, many of the recently fabricated periodic structures possess nonlinear properties that give rise to qualitatively new physical effects such as multistability of a finite nonlinear periodic medium [2] and energy localization in the form of gap solitons [3]. Such effects are usually analyzed in the framework of the coupled-mode theory $[3,4]$, and they are associated with the nonlinearity-dependent tuning of the stop band as the wave intensity is increased.

Recent experiments, such as the observation of optical gap solitons [5] and the control of coherent matter waves in optical lattices [6], as well as theoretical results on gap solitons in Bose-Einstein condensates [7] and the oscillatory instability of Bragg solitons [8], call for a systematic analysis of nonlinear effects in periodic structures beyond the approximation provided by the coupled-mode theory. Such an analysis is crucially important for determining stability of nonlinear waves in periodic structures because the wave instabilities can appear due to the mode coupling to other bands.

In this Letter, we present the first analysis of the existence and stability of nonlinear localized waves in a periodic medium with multiple gaps in the transmission spectrum that is valid beyond the coupled-mode theory. We consider a simplified (but still very general) model where waves are localized in a layered medium by an intensity-dependent defect (or, in other words, they are guided by a thin-layer nonlinear waveguide). Implying the applicability of our results to a variety of different systems, we describe four qualitatively different physical situations and characterize, in the framework of a unified
PACS numbers: 42.70.Qs, 68.65.-k, 74.80.Dm, 75.70.Cn

and systematic approach, the properties of two types of nonlinear localized waves [9]. For the first time to our knowledge, we analyze stability of nonlinear localized waves in a periodic medium and reveal an important physical mechanism of wave instability associated with the band-gap resonances.

Model.-We describe localized waves in a superlattice using the nonlinear Schrödinger equation for the normalized wave envelope $\psi(x, t)$,

$$
i \frac{\partial \psi}{\partial t}+\frac{\partial^{2} \psi}{\partial x^{2}}+\mathcal{F}(I ; x) \psi=0,
$$

where $I \equiv|\psi|^{2}$ is the wave intensity, $t$ is time (or propagation variable), $x$ is the spatial coordinate, and the real function $\mathcal{F}(I ; x)$ describes both nonlinear and periodic properties of the medium. We note that the system (1) is Hamiltonian, and for localized solutions the power $P=$ $\int_{-\infty}^{+\infty} I(x) d x$ is conserved.

We seek stationary localized solutions of Eq. (1) in the form $\psi(x, t)=u(x ; \omega) e^{i \omega t}$, where $\omega$ is the normalized frequency (or the propagation constant, in optics), and the real function $u(x ; \omega)$ satisfies the equation

$$
-\omega u+\frac{d^{2} u}{d x^{2}}+\mathcal{F}(I ; x) u=0 .
$$

We assume that the superlattice is linear, and nonlinearity appears only through the properties of an embedded localized defect. Then, if the corresponding width of the wave envelope is much larger than that of the defect, the inhomogeneity can be modeled by a delta function and, in the simplest case, we can write $\mathcal{F}(I ; x)=\nu(x)+\delta(x) G(I)$, where the function $G(I)$ characterizes the properties of the defect, and $\nu(x) \equiv \nu(x+h)$ describes an effective potential of the superlattice with the spatial period $h$.

For such a nonlinearity, localized waves can be constructed with the help of matching conditions, by using the solutions of Eq. (2) with $\mathcal{F}(I ; x)=\nu(x)$, presented in the form of the Bloch-type wave functions [1].

Band-gap structure and localized waves. - If the effective periodic potential $\nu(x)$ is approximated by a piecewise-constant function (the Kronig-Penney model), the solution can be decomposed into a pair of counterpropagating waves with the amplitudes $a(x ; \omega)$ and $b(x ; \omega)$, 


$$
u_{b}(x ; \omega)=a(x ; \omega) e^{-\mu(x ; \omega) x}+b(x ; \omega) e^{+\mu(x ; \omega) x},
$$

where $\mu(x ; \omega)=\sqrt{\omega-\nu(x)}$ is the local wave number. As follows from the Floquet-Bloch theory, for a Bloch-wave solution the reflection coefficient $r(x ; \omega)=$ $b(x ; \omega) / a(x ; \omega)$ is a periodic function, i.e., $r(x ; \omega)=$ $r(x+h ; \omega)$, and it satisfies the eigenvalue problem:

$$
T(x ; \omega)\left(\begin{array}{c}
1 \\
r(x ; \omega)
\end{array}\right)=\tau(\omega)\left(\begin{array}{c}
1 \\
r(x ; \omega)
\end{array}\right),
$$

where $T(x ; \omega)$ is a transfer matrix that describes a change of the wave amplitudes $\{a, b\}$ after one period $(x, x+h)$ [10]. It was found that $\operatorname{det} T \equiv 1$, and therefore two linearly independent solutions of Eq. (4) correspond to a pair of the eigenvalues $\tau$ and $\tau^{-1}$. Relation $\tau(\omega)$ determines a band-gap structure of the superlattice spectrum: the waves are propagating, if $|\tau|=1$, and they are localized, if $|\tau| \neq 1$. In the latter case, a nonlinear defect can support nonlinear localized waves as bound states of Bloch-wave solutions with the asymptotics $|u(x \rightarrow \pm \infty)| \rightarrow 0$. The wave amplitude at the defect is determined from the continuity condition at $x=0$, i.e., $I_{0} \equiv I\left(0^{+}\right)=I\left(0^{-}\right)$and $[d u / d x]_{x=0^{-}}^{0^{+}}+G\left(I_{0}\right) u_{x=0}=0$. Then, we use Eq. (3) to express the latter condition through the superlattice characteristics,

$$
G_{0} \equiv G\left(I_{0}\right)=\zeta(\omega) .
$$

Here $\zeta=\left(\zeta^{+}+\zeta^{-}\right)_{x=0}, \zeta^{ \pm}=\mu^{ \pm}\left(1-r^{ \pm}\right)\left(1+r^{ \pm}\right)^{-1}$, and "+" and "-" stand for the characteristics on the right and left sides of the defect, respectively, i.e., $\nu(x)=$ $\nu^{+}(|x|)$, for $x>0$, and $\nu(x)=\nu^{-}(|x|)$, for $x<0$.

Relation (5) allows us to identify different types of nonlinear localized states. We notice that such localized states, supported by an attractive nonlinear defect $\left(G_{0}>0\right)$, exist in the so-called waveguiding regime, when $\zeta(\omega)>0$. Additionally, localization can occur at a repulsive nonlinear defect $\left(G_{0}<0\right)$ in the antiwaveguiding regime, provided $\zeta(\omega)<0$.

To study the linear stability, we consider the evolution of small-amplitude perturbations of the localized state presenting the solution in the form

$$
\psi(x, t)=\left\{u(x)+v(x) e^{i \gamma t}+w^{*}(x) e^{-i \gamma^{*} t}\right\} e^{i \omega t},
$$

and obtain the linear eigenvalue problem for $v(x)$ and $w(x)$, which is used to determine the possible eigenvalues $\gamma$. Specifically, from the requirement of mode localization it follows that the eigenvalues should satisfy the matching condition $Y(\gamma)=0[11]$, where $Y(\gamma)=\left[G_{1}-\zeta(\omega+\right.$ $\gamma)]\left[G_{1}-\zeta(\omega-\gamma)\right]-\left(G_{1}-G_{0}\right)^{2}, \quad G_{1} \equiv G_{0}+$ $I_{0} G^{\prime}\left(I_{0}\right)$, and $I_{0}$ is calculated for an unperturbed solution. In general, the eigenmode solutions fall into one of the following categories: (i) internal modes with real eigenvalues describe periodic oscillations ("breathing") of the localized state, (ii) instability modes correspond to purely imaginary eigenvalues with $\operatorname{Im} \gamma<0$, and (iii) oscillatory instabilities can occur when the eigenvalues are complex (and $\operatorname{Im} \gamma<0$ ). Since the linear spectrum has a symmetry $\gamma \rightarrow \pm \gamma^{*}$, eigenmodes with $\operatorname{Im} \gamma \neq 0$ always indicate instability, and in the following we consider only solutions with $\operatorname{Re} \gamma \geq 0$ and $\operatorname{Im} \gamma \geq 0$ with no lack of generality.

To demonstrate the basic stability results, we consider a localized defect possessing a cubic nonlinear response, $G(I)=\alpha+\beta I$. Under proper scaling the absolute value of the nonlinear coefficient $\beta$ can be normalized to unity, so that $\beta=+1$ corresponds to self-focusing and $\beta=-1$ to self-defocusing nonlinearity. Localization depends also on the sign of the linear coefficient $\alpha$, which defines the defect response at small intensities: attractive if $\alpha>0$, and repulsive otherwise. Therefore, below we consider four qualitatively different examples that correspond to different signs of $\alpha$ and $\beta$.

Self-focusing nonlinearity. - First we consider the properties of localized waves supported by a defect with a self-focusing nonlinearity $(\beta=+1)$ and attractive linear response $(\alpha>0)$. Such waves already exist in the linear limit at the frequencies $\omega_{b}$ defined by the equality $\zeta\left(\omega_{b}\right)=\alpha$, and they correspond to the waveguiding regime only (white regions in Fig. 1, top). Using the guided-wave terminology, we notice that the first band gap (semi-infinite white region at $\omega>19$ ) corresponds to the conditions of internal reflection (IR) and, therefore, its
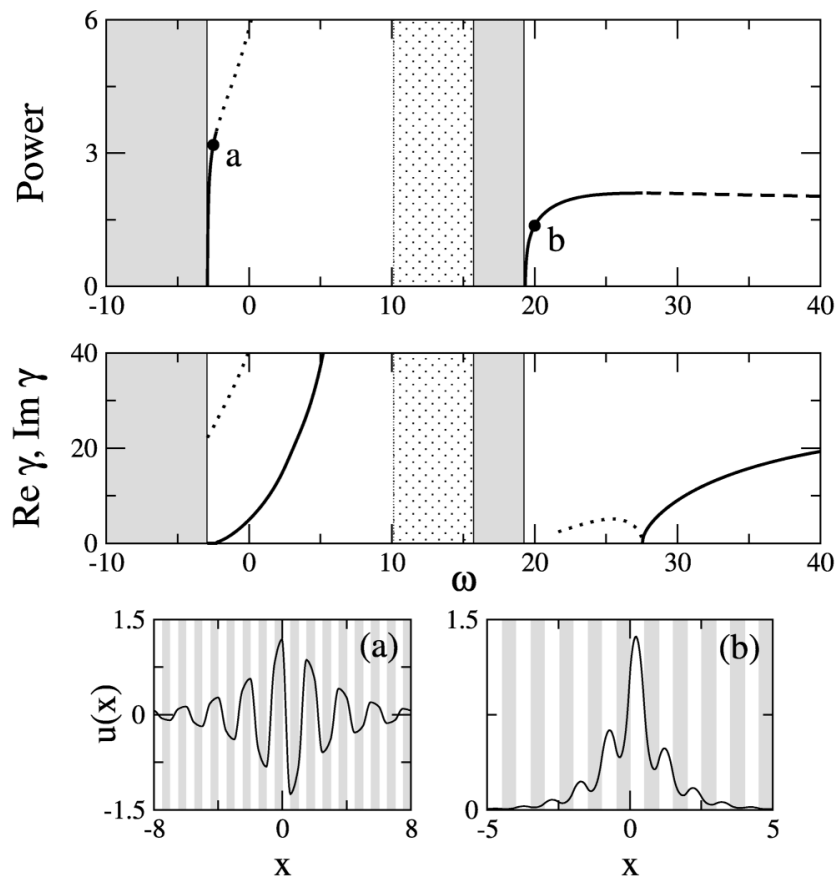

FIG. 1. Top: Power vs frequency dependences for the localized waves: solid line-stable; dashed line-unstable; and dotted line-oscillatory unstable. Middle: real (dotted line) and imaginary (solid line) parts of the eigenvalues associated with the wave instability. Shading marks "waveguiding" (white) and "antiwaveguiding" (dotted) localization regimes inside the band gaps. Bottom: the localized states corresponding to the marked points $(a),(b)$ in the top plot; shading marks the areas with smaller $\nu$. The lattice parameters are $h=1, \nu(x)=0$ for $n-1 / 2<x / h<n$, and $\nu(x)=30$ for $n<x / h<n+1 / 2$, where $n$ is integer, and $\alpha=0.5, \beta=1$. 
dispersion properties should be similar to those of the conventional waveguiding regime. Localized waves in this band resemble ordinary guided waves modulated by a periodic structure [see Fig. 1(b)]. Since an IR wave profile does not contain zeros, it is a fundamental eigenmode. Therefore, the conditions of the Vakhitov-Kolokolov (VK) stability theorem [12] are satisfied, and the IR states are unstable if and only if $d P / d \omega<0$. At the critical point, $d P / d \omega=0$, the linear eigenvalue passes through zero and becomes imaginary, as illustrated in Fig. 1 (middle, $\omega \simeq 27.5$ ).

In contrast, band gaps appear at smaller $\omega$ due to the resonant Bragg reflection (BR) at the periodic structure, so that the localized waves are somewhat similar to gap solitons composed of mutually coupled backward and forward propagating waves [see Fig. 1(a)]. For the BR states, the VK criterion provides only a necessary condition for stability, since the higher-order localized states can also exhibit oscillatory instabilities. Indeed, we notice that in the linear limit there always exists an internal mode corresponding to a resonant coupling between the BR and IR band gaps, since $Y\left(\omega_{b}^{(I R)}-\omega_{b}^{(B R)}\right) \equiv 0$. We perform extensive numerical calculations and find that this mode leads to an oscillatory instability of BR waves when the value $(\omega-\operatorname{Re} \gamma)$ moves outside the band gap; it occurs when the wave intensity exceeds a threshold value (see Figs. 1 and 2).

Next, we study the linearly repulsive defect $(\alpha<0)$ with self-focusing nonlinearity. In this case, the waveguiding-type states bifurcate from the band-gap edges [with $G_{0}=\zeta\left(\omega_{b}\right)=0$ ] and the stability criteria are the same as in the case $\alpha>0$ discussed above; see Fig. 3 (top, white regions), and characteristic mode profiles in Figs. 3(a) and 3(c). The major difference from the previous case is the existence of the antiwaveguiding states at small intensities, $I_{0}<|\alpha / \beta|$; see Fig. 3 (top, dotted region). We find that such waves can be stable at

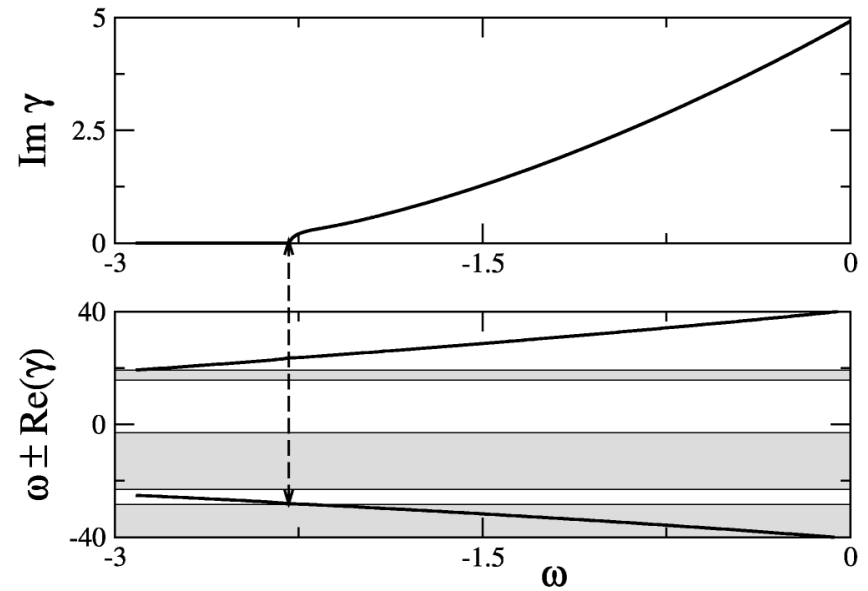

FIG. 2. An example of a resonance that occurs between an internal mode of the localized wave and a bang-gap edge and leads to an oscillatory instability. low powers in the lowest-order band gap [such as one shown in Fig. 3(b)], while at higher powers such states exhibit oscillatory instabilities due to a resonance with higher-frequency bands.

Self-defocusing nonlinearity.-Let us now consider the case $\alpha<0$ and $\beta=-1$, when the total response of the defect is negative for any intensity, i.e., $G(I)<0$. In this case, the localized waves can exist only in the antiwaveguiding regime [see Fig. 4(a), dotted regions], the nonlinear waves continue the linear impurity states, and their frequency decreases at higher powers. We find that the localized states corresponding to the lowest BR gap (at larger $\omega$ ), are always stable [note, however, that instabilities can appear if the superlattice parameters are different]. However, in other band gaps the localized states can exhibit oscillatory instability due to a resonance with the lower bands.

Finally, we study the case of a linearly attractive defect $(\alpha>0)$ possessing a self-defocusing nonlinearity $(\beta=$ $-1)$. At small intensities, the defect is attractive, and it can support localized waves in a waveguiding regime, in both IR and BR gaps, as shown in Fig. 4(b) (white regions). Performing the stability analysis, we find that IR waves are always stable, while oscillatory instabilities appear for higher band-gap states. Since at higher intensities, i.e., for $I_{0}>|\alpha / \beta|$, the defect response changes its sign, a new type of localized waves can exist in the antiwaveguiding dispersion region, bifurcating from the band-gap edge, as shown in Fig. 4(b) (dotted region). It is possible to demonstrate that for such waves the VK stability criterion becomes inverted, i.e., the waves are unstable if $d P / d \omega>0$. This happens because the signs of both the nonlinear response and effective dispersion are altered compared to the IR waves supported by a self-focusing defect. All higher band-gap states exhibit oscillatory instabilities.

In conclusion, we have analyzed the existence and stability of all possible types of nonlinear localized waves
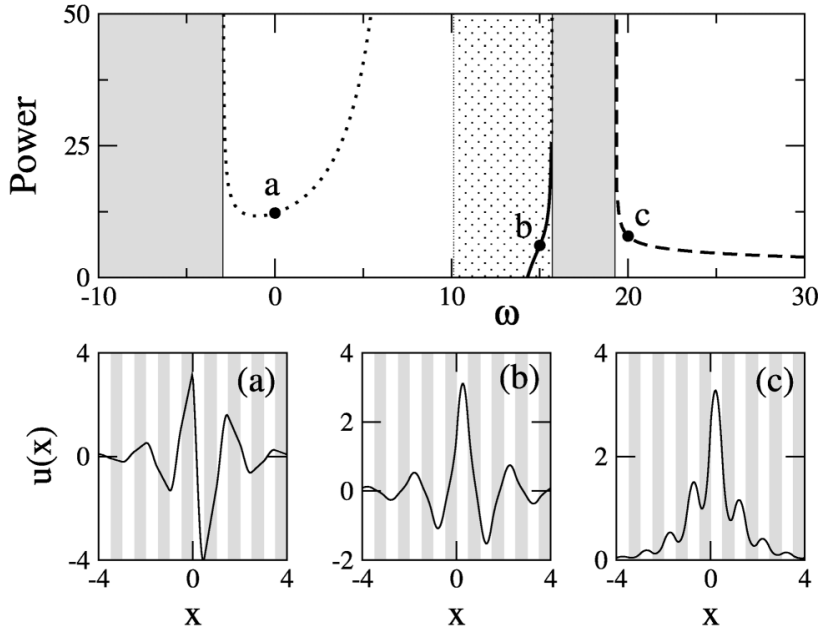

FIG. 3. Power vs frequency, and (a) -(c) wave profiles for $\alpha=$ $-5, \beta=1$. Notations are the same as in Fig. 1 . 

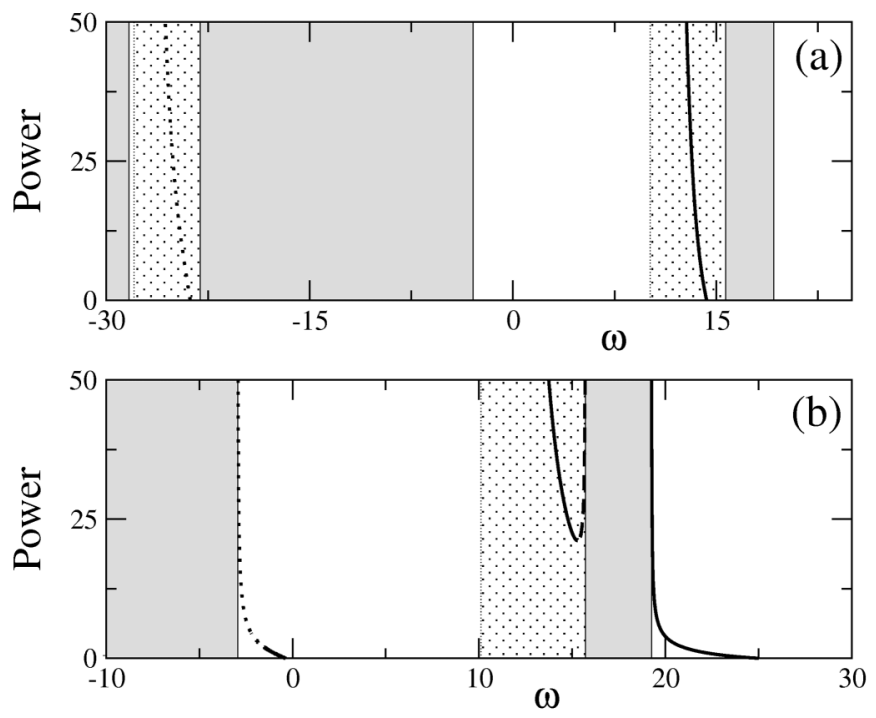

FIG. 4. Power vs frequency, for $\beta=-1$ and (a) $\alpha=-5$, (b) $\alpha=5$. Notations are the same as in Fig. 1 .

in one-dimensional periodic structures considering the simplest nonlinear generalization of the Kronig-Penney model with a localized nonlinearity for which both the wave classification and stability analysis can be carried out in a complete and systematic way. Similar results for the self-trapped states and solitary waves have been recently obtained for a periodic array of nonlinear waveguides [13]. Additionally, many of these results are valid for more realistic physical models of extended nonlinear periodic media. Such cases include the electron self-trapping and locking states in cuprates and semiconductor superlattices [14]; nonlinear guided waves in optical superlattices [15] and waveguide arrays [16]; impurity modes in photonic band-gap materials [17], magneto-optical periodic structures [18], and photonic crystal fibers [19]; coherent matter waves and gap solitons in optical lattices [7,20], etc. In particular, the stability of nonlinear localized impurity modes is a crucial issue for creating tunable band-gap materials where gaps could be controlled by changing the input light intensity. Since the physical mechanism of the resonance-induced instability is generic, we expect that our results will be useful for the further understanding of 2D and 3D models.

We thank O. Bang and C. M. Soukoulis for useful collaboration, S. John for his interest in this work, and also acknowledge support from the Performance and Planning Fund of the Institute of Advanced Studies.
[1] See, e.g., P. Yeh, Optical Waves in Layered Media (John Wiley \& Sons, New York, 1988).

[2] F. Delyon et al., Phys. Rev. Lett. 57, 2010 (1986); D. Hennig and G. P. Tsironis, Phys. Rep. 307, 333 (1999).

[3] Yu. I. Voloshchenko et al., Zh. Tekh. Fiz. 51, 902 (1981) [Sov. Phys. Tech. Phys. 26, 541 (1981)]; W. Chen and D. L. Mills, Phys. Rev. Lett. 58, 160 (1987); D. N. Christodoulides and R. I. Joseph, Phys. Rev. Lett. 62, 1746 (1989); N. Aközbek and S. John, Phys. Rev. E 57, 2287 (1998).

[4] H. Kogelnik and C. V. Shank, J. Appl. Phys. 42, 2327 (1972); H. G. Winful, Appl. Phys. Lett. 46, 527 (1985).

[5] B. J. Eggleton et al., Phys. Rev. Lett. 76, 1627 (1996).

[6] B. P. Anderson and M. A. Kasevich, Science 282, 1686 (1998).

[7] O. Zobay et al., Phys. Rev. A 59, 643 (1999).

[8] I. V. Barashenkov et al., Phys. Rev. Lett. 80, 5117 (1998); A. De Rossi et al., Phys. Rev. Lett. 81, 85 (1998).

[9] In optics, such two types of localized waves correspond to the conventional guided waves, due to the total internal reflection, and to the band-gap states, due to the Bragg-type reflection.

[10] A transfer matrix approach for optical waves was developed by F. Abelès, Ann. Phys. (Paris) 5, 596 (1950); 5, 706 (1950); see also A. A. Sukhorukov et al., Phys. Rev. E 63, 016615 (2001), where notations correspond to Eq. (4).

[11] We notice that $Y(\gamma)$ is also known as the Evans function, see R. L. Pego and M. I. Weinstein in Differential Equations with Applications to Mathematical Physics, edited by W. F. Ames et al. (Academic Press, San Diego, 1993).

[12] N. G. Vakhitov and A. A. Kolokolov, Izv. Vyssh. Uchebn. Zaved. Radiofiz. 16, 1020 (1973) [Radiophys. Quantum Electron. 16, 783 (1973)].

[13] A. A. Sukhorukov and Yu. S. Kivshar, nlin.PS/0105073.

[14] J. Hader, P. Thomas, and S. W. Koch, Progr. Quantum Electron. 22, 123 (1998); Q. Tian and C. Wu, Phys. Lett. A 262, 83 (1999); F. V. Kusmartsev and H. S. Dhillon, Phys. Rev. B 60, 6208 (1999).

[15] Y. Y. Zhu and N. B. Ming, Opt. Quantum Electron. 31, 1093 (1999); A. Saher Helmy, Opt. Lett. 25, 1370 (2000).

[16] H. S. Eisenberg et al., Phys. Rev. Lett. 85, 1863 (2000).

[17] Qiming Li et al., Phys. Rev. B 53, 15577 (1996); A. Mekis et al., Phys. Rev. B 58, 4809 (1998); O. Painter et al., Science 284, 1819 (1999).

[18] See, e.g., M. Inoue et al., J. Appl. Phys. 83, 6768 (1998).

[19] R. F. Cregan et al., Science 285, 1537 (1999); B. J. Eggleton et al., Opt. Lett. 24, 1460 (1999).

[20] F. Barra et al., Phys. Rev. E 61, 5852 (2000); K.-P. Marzlin and W. Zhang, Eur. Phys. J. D 12, 241 (2000); J. C. Bronski et al., Phys. Rev. Lett. 86, 1402 (2001); A. Trombettoni and A. Smerzi, Phys. Rev. Lett. 86, 2353 (2001). 